\title{
Outcome of children relapsing after first allogeneic haematopoietic stem cell transplantation for acute myeloid leukaemia: a retrospective I-BFM analysis of 333 children
}

\author{
Theodor Uden, ${ }^{1}$ iD Alice Bertaina, ${ }^{2}$ \\ Jonas Abrahamsson, ${ }^{3}$ Marc Ansari, \\ Adriana Balduzzi, ${ }^{5}$ \\ Jean-Pierre Bourquin, ${ }^{6}$ \\ Corinne Gerhardt, ${ }^{7}$ Marc Bierings, ${ }^{7}$ \\ Henrik Hasle, ${ }^{8}$ Arjan Lankester, ${ }^{9}$ \\ Kirsten Mischke, ${ }^{1}$ Andrew S. Moore, ${ }^{10}$ \\ Ian Nivison-Smith, ${ }^{11}$ Anna Pieczonka, ${ }^{12}$ \\ Christina Peters, ${ }^{13}$ Petr Sedlacek, ${ }^{14}$ \\ Dirk Reinhardt, ${ }^{15}$ Jerry Stein, ${ }^{16}$ \\ Birgitta Versluys, ${ }^{17}$ Jacek Wachowiak, ${ }^{12}$ \\ Leen Willems, ${ }^{18}$ Martin Zimmermann, ${ }^{1}$ \\ Franco Locatelli ${ }^{2,19}$ (D) and \\ Martin G. Sauer ${ }^{1}$ \\ ${ }^{1}$ Division of Pediatric Hematology and \\ Oncology, Department of Pediatrics, \\ Universities of Medical University \\ Hannover, Hannover, Germany, ${ }^{2}$ IRCCS \\ "Bambino Gesù" Children's Hospital, \\ Rome, Italy, ${ }^{3}$ Institution of Clinical \\ Sciences, Sahlgrenska University Hospital, \\ Gothenburg, Sweden, ${ }^{4}$ Cansearch Research \\ Laboratory, Geneva University Hospital, \\ Geneva University, Geneva, Switzerland, \\ ${ }^{5}$ Clinica Pediatrica Università degli Studi \\ di Milano Bicocca, Fondazione MBBM/ \\ Ospedale San Gerardo, Monza, Italy, \\ ${ }^{6}$ University Children's Hospital Zurich, \\ CHE, Zurich, Switzerland, ${ }^{7}$ Princess \\ Maxima Centre for Pediatric Oncology, \\ Childrens University Hospital Utrecht, \\ Utrecht, Netherlands, ${ }^{8}$ Aarhus University \\ Hospital, Arhus, Sweden, ${ }^{9}$ Willem \\ Alexander Children's Hospital, Leiden \\ University Medical Centre, Leiden, \\ Netherlands, ${ }^{10}$ Queensland Children's \\ Hospital and The University of \\ Queensland, Brisbane, QLD, ${ }^{11}$ Australasian \\ Bone Marrow Transplant Recipient \\ Registry, Darlinghurst, NSW, Australia, \\ ${ }^{12}$ University of Medical Sciences, Poznan, \\ Poland, ${ }^{13}$ St Anna Childrens Hospital, \\ Vienna, Austria, ${ }^{14}$ University Hospital
}

\begin{abstract}
Summary
Outcome of 333 children with acute myeloid leukaemia relapsing after a first allogeneic haematopoietic stem cell transplantation was analyzed. Four-year probability of overall survival (4y-pOS) was 14\%. 4y-pOS for 122 children receiving a second haematopoietic stem cell transplantation was $31 \%$ and $3 \%$ for those that did not $(P=<0 \cdot 0001)$. Achievement of a subsequent remission impacted survival $(P=<0 \cdot 0001)$. For patients receiving a second transplant survival with or without achieving a subsequent remission was comparable. Graft source (bone marrow vs. peripheral blood stem cells, $P=0.046$ ) and donor choice (matched family vs. matched unrelated donor, $P=0.029)$ positively impacted survival after relapse. Disease recurrence and non-relapse mortality at four years reached $45 \%$ and $22 \%$.
\end{abstract}

Keywords: allogeneic hematopoietic stem cell transplantation, children, relapse, acute myeloid leukemia, second hematopoietic stem cell transplantation. 
Motol, Charles University, Prague, Czech

Republic, ${ }^{15}$ University of Duisburg-Essen, Essen, Germany, ${ }^{16}$ Schneider Children's

Medical Center of Israel, Petach Tikva, Israel, ${ }^{17}$ University Medical Center Utrecht, Utrecht, Netherlands, ${ }^{18}$ Ghent University Hospital, Ghent, Belgium and ${ }^{19}$ Sapienza, University of Rome, Rome, Italy

Received 26 August 2019; accepted for

publication 28 October 2019

Correspondence: Martin G. Sauer, Department of

Pediatric Hematology and Oncology, Medizinische

Hochschule Hannover, OE 6780, Carl-Neuberg-

Strasse 1, 30625 Hannover, Germany.

E-mail: sauer.martin@mh-hannover.de

Haematopoietic stem cell transplantation (HSCT) has contributed substantially to the cure of children with high-risk acute myeloid leukaemia (AML) (Kaspers et al., 2013; Rasche et al., 2018). Nevertheless, relapse after HSCT remains the main reason for treatment failure (Pession et al., 2013; O'Hare et al., 2017). Here, we retrospectively identified 333 children and adolescents from 15 countries that had experienced AML relapse after a first allogeneic HSCT.

\section{Patients and methods}

A retrospective data analysis across national study groups participating in the I-BFM consortium was performed. A questionnaire was submitted to the representatives of each of the national study groups that had accepted to participate.

Relapse was defined as cytological proof of leukaemia in the bone marrow, central nervous system or other extramedullary sites. Complete remission was defined as the presence of normal haematopoiesis and $\leq 5 \%$ blasts in the bone marrow. Patients with $\mathrm{t}(8 ; 21), \mathrm{t}(15 ; 17)$ or $\operatorname{inv}(16)$ were defined to be standard-risk (Grimwade et al., 2010).

Probabilities of OS were estimated according to the Kaplan-Meier method and comparisons were done with the log-rank test (Kaplan \& Meier, 1958). A Cox regression analysis was performed to identify predictors of survival. Treatment- and disease-related mortality were calculated as cumulative incidences according to Kalbfleisch and Prentice (Kalbfleisch \& Sprott, 1974; Prentice et al., 1978). Multivariate analysis was performed for risk factors reaching a $P$ value $<0 \cdot 1$ in univariate analysis.

\section{Results}

\section{Patient characteristics}

For this study, 333 children were identified with a median follow-up of $22 \cdot 1$ months (range $0-60) ; 122$ (36.6\%) of these patients had a second HSCT. Median age was 8.5 years. There were $176 / 309$ patients $(57 \cdot 0 \%)$ younger than 10 years and 198/333 (59.5\%) children were male. Information on cytogenetic lesions was available for 209 children. Of them, $180(86 \cdot 1 \%)$ were stratified as high -risk (HR) and 29 (13.9\%) as standard -risk (SR) according to the definition given above. Of 326 patients with known remission status at first HSCT, $156(47.9 \%)$ had been transplanted in first complete remission (CR1), 145 (44.5\%) in second complete remission (CR2) and $25(7.7 \%)$ did not achieve remission (NR). Median time between first HSCT and relapse was 6.64 months (Q1: 3.6, Q3: 12.4). Among the 181 patients for whom information was available, a subsequent remission after post-HSCT relapse was achieved in 74 patients $(40 \cdot 9 \%)$. Information on whether the same or a different donor was used for the second HSCT was available for 15 patients only.

\section{Total outcome}

The probability of overall survival at four years for the total cohort was $14 \%$ (standard error, SE $=0.02$, Fig 1A). HSCT in CR1 (4y-pOS 14\%) versus CR2 (4y-pOS 16\%) did not impact survival. Achievement of a subsequent remission was important (4y-pOS $33 \%$ vs. $5 \%$, Fig 1B).

Time between first HSCT and relapse ( $<6$ months) was associated with inferior $4 y$-pOS $(6 \%$ vs. $21 \%, P<0.0001$, Fig 1C). This was confirmed in multivariate analysis [(relapse $\geq 6$ months after first HSCT): HR, 0.58; 95\% CI, 0.41-0.83; $P=0 \cdot 003]$.

Overall survival was comparable for children originally presenting with SR versus HR cytogenetic lesions, both for the total cohort (4y-pOS 13\% for HR vs. $4 y$-pOS $13 \%$ for SR; $P=0 \cdot 71$ ), and those proceeding to a second HSCT (4ypOS $28 \%$ for HR vs. $4 y$-pOS $40 \%$ for SR; $P=0 \cdot 31$ ).

Neither age ( $<10$ years vs. $\geq 10$ years; $P=0 \cdot 38)$, nor gen$\operatorname{der}(P=0 \cdot 20)$ were relevant. 
(A)

$4 y$-pOS for the total cohort

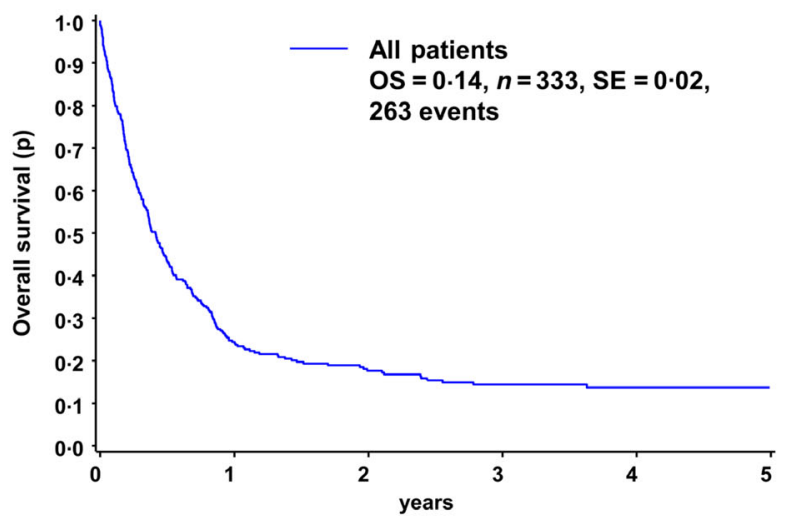

(C) Impact of the interval between 1st HSCT and relapse on 4y-pOS

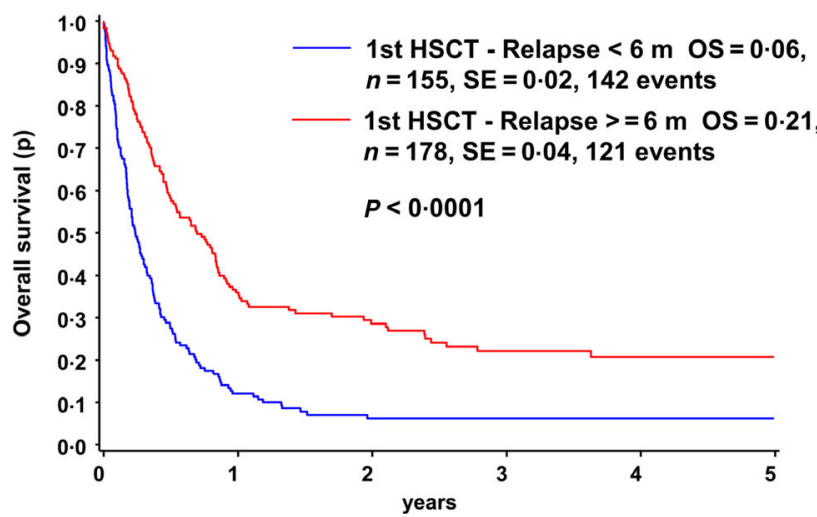

(B) 4y-pOS depending on the achievement of another remission

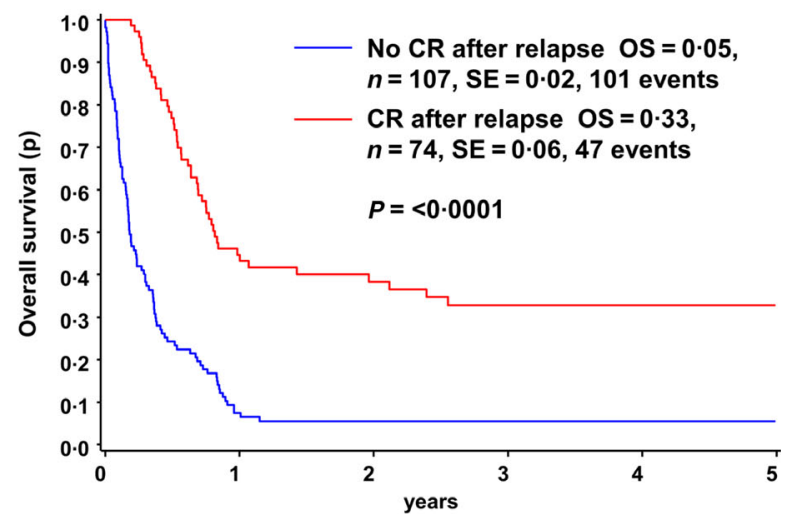

(D) $\quad 4 y-p O S$ with or without a 2nd HSCT

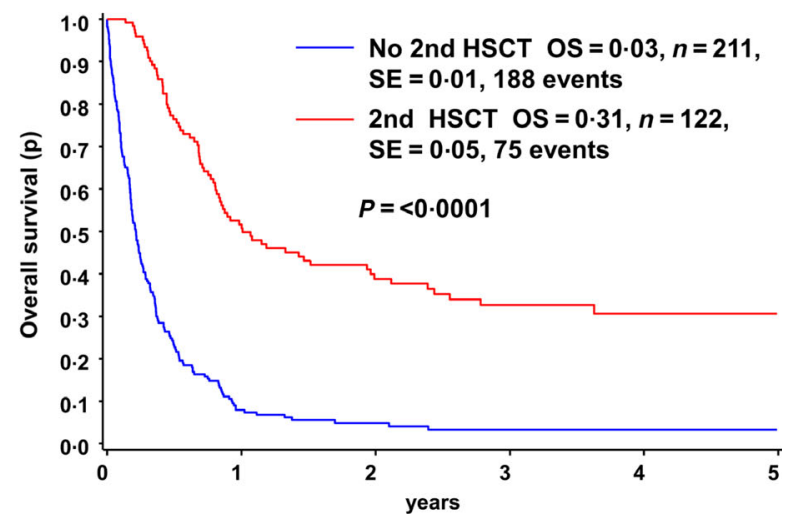

Fig 1. Overall survival for children relapsing after a first allo-HSCT [four-year probability of overall survival (pOS)]. CR, complete remission; HSCT, haematopoietic stem cell transplantation.

\section{Outcome after second HSCT}

The 4y-pOS for the 122 children proceeding to a second HSCT was $31 \%$ and $3 \%$ for those that did not $(P<0.0001$, Fig 1D). In all, 55/74 children (74.3\%) achieved another remission; 19 had residual disease. For the latter survival was similar to that of patients who achieved a subsequent remission (4y-pOS $31 \%$ with no remission vs. $4 y$-pOS $39 \%$ with remission; $P=0 \cdot 45$; Fig 2A). Second HSCTs were performed at a median of 2.9 months (Q1: 2.1, Q3: 3.8) after relapse and were an independent prognostic factor in multivariate analysis [(second HSCT performed): HR, 0.27; 95\% CI, $0 \cdot 18-0 \cdot 41 ; P=<0 \cdot 001]$.

Poor performance prevented a second HSCT for $10.5 \%$ of children only. Major reasons were insufficient disease control (55.2\%). Parental choice, early decision for palliative care or not further specified reasons accounted for $34 \cdot 3 \%$.

Graft sources for second HSCT were bone marrow (BM, 43/119 patients, 36.1\%), peripheral blood stem cells (PBSC, 65/119 patients, 54.6\%), and umbilical cord blood (CB, 11/ 119 patients, 9.2\%). Matched unrelated donors (MUDs) were used for the majority of children (53/114 patients, $46 \cdot 5 \%$ ), matched family donors (MFDs) for 28/114 (24.6\%), partially (p-)MFDs for $17 / 114$ (14.9\%), and haplo donors for $16 / 114$ (14.0\%) patients.

Bone marrow was associated with significantly better survival in case the second HSCT was done $\geq 6$ months after relapse [ 4 y-pOS $56 \%$ for BM vs. $4 y$-pOS $44 \%$ for CB vs. $4 \mathrm{y}$-pOS $28 \%$ for PBSC, $P(\mathrm{BM}$ vs. $\mathrm{PBSC})=0.046$, Fig $2 \mathrm{~B}]$.

Children receiving their second transplant from a MFD (4y-pOS 44\%) fared better than MUD [4y-pOS 32\%, $P$ (MFD vs. MUD) $=0.029$ ] or pMFD recipients [4y-pOS $15 \%, P$ $(\mathrm{MFD}$ vs. $\mathrm{pMFD})=0.0023$, Fig $2 \mathrm{C}$, multivariate analysis $[$ donor type $=\mathrm{MFD}]: \quad \mathrm{HR}, \quad 0.42 ; \quad 95 \% \quad \mathrm{CI}, \quad 0.21-0.82$; $P=0.011]$. Outcome for MUD transplants improved after 2010 (4y-pOS 18\% for HSCT before 2010 vs. 4y-pOS $41 \%$ for HSCT in or after 2010; $P=0.045$; Fig 2D).

With a four-year cumulative incidence (4y-CI) of $45 \%$, relapse remained the main reason for treatment failure. The $4 y-C I$ of transplant-related mortality (TRM) was $22 \%(9 \%$ for MFDs, $14 \%$ for haplo donors, $28 \%$ for MUDs, and $38 \%$ for pMFD $)[P($ MFD vs. $\mathrm{MUD})=0.016, \quad P(\mathrm{MFD}$ vs. pMFD) $=0 \cdot 018$. 
(A) Impact of remission status prior to 2nd HSCT on 4y-pOS

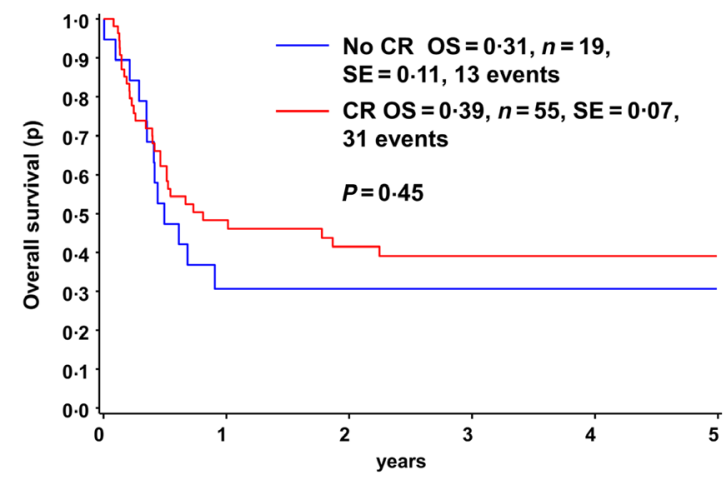

(C)

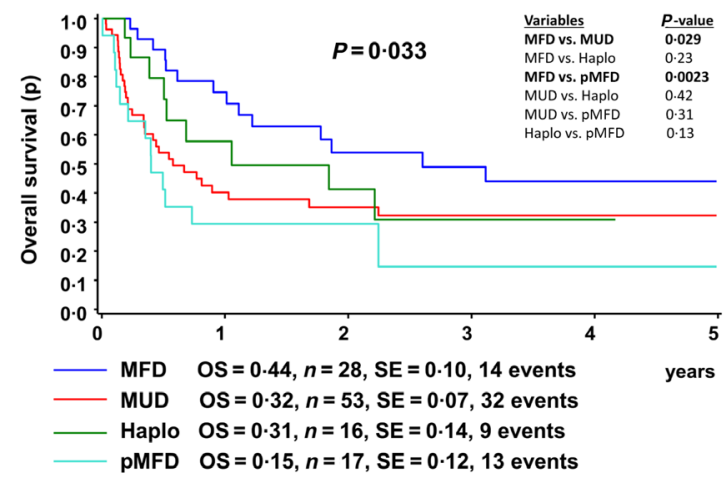

(B) Impact of graft source and interval between relapse and 2nd HSCT on 4y-pOS

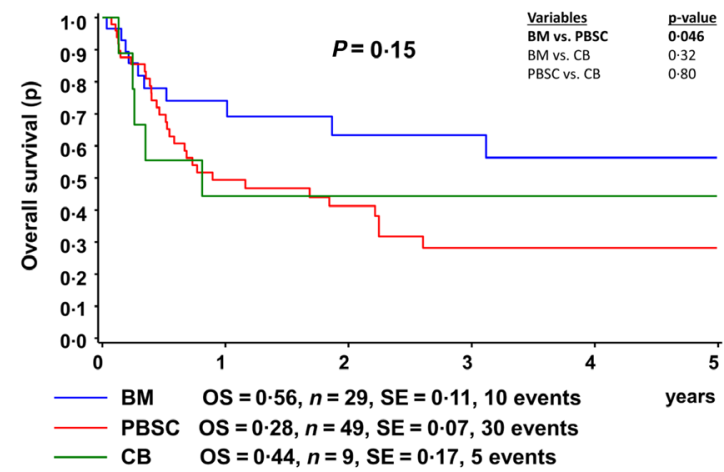

(D) 4y-pOS after 2nd HSCTs from a MUD: Before and after 2010

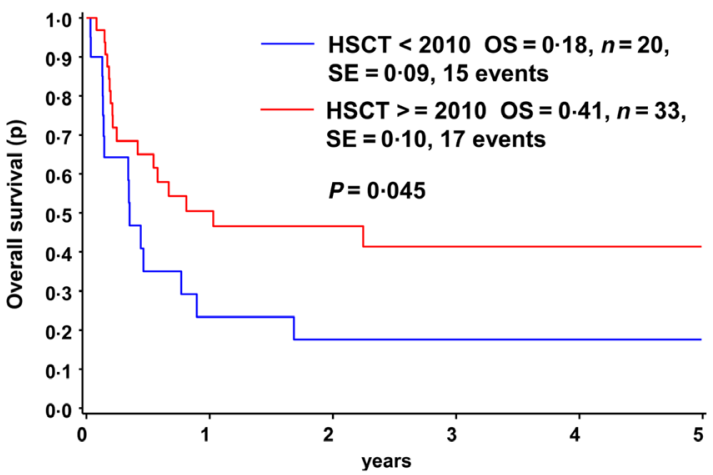

Fig 2. Overall survival for patients that received a second allo-HSCT [four-year- probability of overall survival (pOS)]. CB, cord blood; CR, complete remission; HSCT, haematopoietic stem cell transplantation; MFD, matched family donor; MSD, matched sibling donor; MUD, matched unrelated donor; Haplo, haploidentical donor; PBSC, peripheral blood stem cells; pMFD, partially matched family donor.

\section{Discussion}

Leukaemic relapse after HSCT has been repeatedly addressed by several groups, but several aspects remain unsatisfactory. First, several larger cohorts combine adult and paediatric patients. Since these cohorts are dominated by adult patients this might lead to conclusions being less relevant for children (Christopeit et al., 2013; Bejanyan et al., 2015; Ruutu et al., 2015; Orti et al., 2016). Second, collecting these patients over very long time intervals pools patients from eras in which risk stratification for transplant, donor HLA-typing technologies, graft-versus-host disease prophylaxis and supportive care differ substantially (Bejanyan et al., 2015). Third, the majority of larger paediatric analyses on second HSCTs combine acute lymphocytic leukaemia (ALL) and AML relapses (Menon et al., 2016; Roux et al., 2017; Yaniv et al., 2018; Lund et al., 2019). In regard of the potentially higher susceptibility of AML to graft versus leukaemia effects and the decisively defined role of minimal residual disease (MRD) before HSCT in ALL (Bader et al., 2002), it might be relevant to do this analysis separately.

The most important reason for treatment failure after a second HSCT remained disease recurrence and TRM. Most
TRM events occurred during the first year whereas relapses plateaued after $2-3$ years. This was comparably reported by a recent US American combined cohort of paediatric ALL and AML children (Lund et al., 2019).

Remission status prior to second HSCT was classified in a dichotomous manner (yes/no) according to the local centre's morphological evaluation. For those children finally transplanted after re-induction without achieving remission, survival at four years surprisingly reached $31 \%$. Although numbers are low, this might reflect an important difference to ALL patients in whom achieving a deep molecular remission before transplant has shown to be a prerequisite for leukaemia-free survival (Bader et al., 2002).

As reported by others, the interval between first HSCT and relapse was a strong predictor for survival (Bosi et al., 2001, Eapen et al., 2004; Ruutu et al., 2015). Notably, Lund et al. (2019) presented an analysis contradicting this. They identified the interval between relapse and second HSCT as being more relevant.

For our analysis, information on whether the same or an alternative donor was used for the second HSCT could be obtained for 15 children only. A series of studies conclude that recruiting a different donor than the one used for the 
first transplant was not advantageous (Christopeit et al., 2013; Ruutu et al., 2015).

Consistent with previous reports, the use of BM versus PBSC had no significant impact on survival (Guardiola et al., 2000; Lund et al., 2019). Nevertheless, after late relapses the use of BM seemed beneficial.

Poor performance status in children has recently been reported to negatively impact outcome after first HSCT (Bitan et al., 2014). Of interest, poor performance status was indicated as a reason for not performing a second HSCT in $10 \cdot 5 \%$ of our cohort only.

Data on the conditioning regimen used were not available for all children. The majority received a myeloablative total body irradiation-free preparative regimen for their second HSCT. Previous studies did not find an association between the intensity of conditioning with relapse or OS (Yaniv et al., 2018; Lund et al., 2019). When differences were reported this might have been attributed to the inclusion of adults (Ruutu et al., 2015).

Despite the fact that this analysis, to our knowledge, represents the largest cohort of children experiencing AML relapse after a first HSCT, our study has clear limitations in terms of its retrospective nature and limited access to more specific transplant-relevant information. With a certain degree of caution, however, some conclusions might be drawn. First, without a second HSCT, there is currently almost no chance for survival. Second, a second HSCT is often not performed despite disease control and acceptable performance status, and third, even for some patients with residual disease a second HSCT might be a relevant treatment option.

\section{Conflicts of interest}

The authors declare no competing financial interests.

\section{References}

Bader, P., Hancock, J., Kreyenberg, H., Goulden, N.J., Niethammer, D., Oakhill, A., Steward, C.G., Handgretinger, R., Beck, J.F. \& Klingebiel, T. (2002) Minimal residual disease (MRD) status prior to allogeneic stem cell transplantation is a powerful predictor for post-transplant outcome in children with ALL. Leukemia, 16, 1668 1672.

Bejanyan, N., Weisdorf, D.J., Logan, B.R., Wang, H.L., Devine, S.M., de Lima, M., Bunjes, D.W. \& Zhang, M.J. (2015) Survival of patients with acute myeloid leukemia relapsing after allogeneic hematopoietic cell transplantation: a center for international blood and marrow transplant research study. Biology of Blood and Marrow Transplantation: Journal of the American Society for Blood and Marrow Transplantation, 21, 454-459.

Bitan, M., He, W., Zhang, M.J., Abdel-Azim, H., Ayas, M.F., Bielorai, B., Carpenter, P.A., Cairo, M.S., Diaz, M.A., Horan, J.T., Jodele, S., Kitko, C.L., Schultz, K.R., Kletzel, M., Kasow, K.A., Lehmann, L.E., Mehta, P.A., Shah, N., Pulsipher, M.A., Prestidge, T., Seber, A., Shenoy, S., Woolfrey, A.E., Yu, L.C. \& Davies, S.M. (2014) Transplantation for children with acute myeloid leukemia: a comparison of outcomes with reduced intensity and myeloablative regimens. Blood, 123, 1615-1620.

Bosi, A., Laszlo, D., Labopin, M., Reffeirs, J., Michallet, M., Gluckman, E., Alessandrino, P.E., Locatelli, F., Vernant, J.P., Sierra, J., Jouet, J.P., Frassoni, F. \& Acute Leukemia Working Party of the European Blood and Marrow Transplant Group (2001) Second allogeneic bone marrow transplantation in acute leukemia: results of a survey by the European Cooperative Group for Blood and Marrow Transplantation. Journal of Clinical Oncology, 19, 3675-3684.

Christopeit, M., Kuss, O., Finke, J., Bacher, U., Beelen, D.W., Bornhauser, M., Schwerdtfeger, R., Bethge, W.A., Basara, N., Gramatzki, M.,
Tischer, J., Kolb, H.J., Uharek, L., Meyer, R.G., Bunjes, D., Scheid, C., Martin, H., Niederwieser, D., Kroger, N., Bertz, H., Schrezenmeier, H. \& Schmid, C. (2013) Second allograft for hematologic relapse of acute leukemia after first allogeneic stem-cell transplantation from related and unrelated donors: the role of donor change. Journal of Clinical Oncology, 31, 3259-3271.

Eapen, M., Giralt, S.A., Horowitz, M.M., Klein, J.P., Wagner, J.E., Zhang, M.J., Tallman, M.S., Marks, D.I., Camitta, B.M., Champlin, R.E., Ringden, O., Bredeson, C.N., Martino, R., Gale, R.P., Cairo, M.S., Litzow, M.R. \& Delima, M. (2004) Second transplant for acute and chronic leukemia relapsing after first HLA-identical sibling transplant. Bone Marrow Transplantation, 34, 721-727.

Grimwade, D., Hills, R.K., Moorman, A.V., Walker, H., Chatters, S., Goldstone, A.H., Wheatley, K., Harrison, C.J., Burnett, A.K. \& National Cancer Research Institute Adult Leukaemia Working Group (2010) Refinement of cytogenetic classification in acute myeloid leukemia: determination of prognostic significance of rare recurring chromosomal abnormalities among 5876 younger adult patients treated in the United Kingdom Medical Research Council trials. Blood, 116, 354-365.

Guardiola, P., Kuentz, M., Garban, F., Blaise, D., Reiffers, J., Attal, M., Buzyn, A., Lioure, B., Bordigoni, P., Fegueux, N., Tanguy, M.L., Vernant, J.P., Gluckman, E. \& Socie, G. (2000) Second early allogeneic stem cell transplantations for graft failure in acute leukaemia, chronic myeloid leukaemia and aplastic anaemia. French Society of Bone Marrow Transplantation. British Journal of Haematology, 111, 292-302.

Kalbfleisch, J.G. \& Sprott, D.A. (1974) Statistical analysis of data bearing on the number of particles required to form a plaque. The Journal of Hygiene, 73, 27-34.

Kaplan, E.L. \& Meier, P. (1958) Nonparametric-estimation from incomplete observations. Journal of the American Statistical Association, 53, 457481.

Kaspers, G.J., Zimmermann, M., Reinhardt, D., Gibson, B.E., Tamminga, R.Y., Aleinikova, O., Armendariz, H., Dworzak, M., Ha, S.Y., Hasle, H., Hovi, L., Maschan, A., Bertrand, Y., Leverger, G.G., Razzouk, B.I., Rizzari, C., Smisek, P., Smith, O., Stark, B. \& Creutzig, U. (2013) Improved outcome in pediatric relapsed acute myeloid leukemia: results of a randomized trial on liposomal daunorubicin by the International BFM Study Group. Journal of Clinical Oncology, 31, 599-607.

Lund, T.C., Ahn, K.W., Tecca, H.R., Hilgers, M.V., Abdel-Azim, H., Abraham, A., Diaz, M.A., Badawy, S.M., Broglie, L., Brown, V., Dvorak, C.C., Gonzalez-Vicent, M., Hashem, H., Hayashi, R.J., Jacobsohn, D.A., Kent, M.W., Li, C.K. Margossian, S.P., Martin, P.L., Mehta, P., Myers, K., Olsson, R., Page, K., Pulsipher, M.A., Shaw, P.J., Smith, A.R., Triplett, B.M., Verneris, M.R. \& Eapen, M. (2019) Outcomes after second hematopoietic cell transplantation in children and young adults with relapsed acute leukemia. Biology of Blood and Marrow Transplantation: Journal of the American Society for Blood and Marrow Transplantation, 25, 301-306.

Menon, N.N., Jenkins, L.M., Cui, H., Jenkins, C., Anwer, F., Yeager, A.M. \& Katsanis, E. (2016) Factors associated with improved outcomes after second allogeneic hematopoietic cell transplantation for relapsed pediatric leukemia. Annals of Hematology, 95, 637-644.

O’Hare, P., Lucchini, G., Cummins, M., Veys, P., Potter, M., Lawson, S., Vora, A., Wynn, R., Peniket, A., Kirkland, K., Pearce, R., Perry, J. \& Amrolia, P.J. (2017) Allogeneic stem cell transplantation for refractory acute myeloid leukemia in pediatric patients: the UK experience. Bone Marrow Transplantation, 52, 825-831.

Orti, G., Sanz, J., Bermudez, A., Caballero, D., Martinez, C., Sierra, J., Cabrera Marin, J.R., Espigado, I., Solano, C., Ferra, C., GarciaNoblejas, A., Jimenez, S., Sampol, A., Yanez, 


\section{Short Report}

L., Garcia-Gutierrez, V., Pascual, M.J., Jurado, M., Moraleda, J.M., Valcarcel, D., Sanz, M.A., Carreras, E. \& Duarte, R.F. (2016) Outcome of second allogeneic hematopoietic cell transplantation after relapse of myeloid malignancies following allogeneic hematopoietic cell transplantation: a retrospective cohort on behalf of the Grupo Espanol de Trasplante Hematopoyetico. Biology of Blood and Marrow Transplantation: Journal of the American Society for Blood and Marrow Transplantation, 22, 584-588.

Pession, A., Masetti, R., Rizzari, C., Putti, M.C., Casale, F., Fagioli, F., Lu Ciani, M., Lo Nigro, L., Menna, G., Micalizzi, C., Santoro, N., Testi, A.M., Zecca, M., Biondi, A., Pigazzi, M., Rutella, S., Rondelli, R., Basso, G., Locatelli, F. \& AIEOP Study Group (2013) Results of the AIEOP AML 2002/01 multicenter prospective trial for the treatment of children with acute myeloid leukemia. Blood, 122, 170-178.
Prentice, R.L., Kalbfleisch, J.D., Peterson Jr., A.V., Flournoy, N., Farewell, V.T. \& Breslow, N.E (1978) The analysis of failure times in the presence of competing risks. Biometrics, 34, 541-554.

Rasche, M., Zimmermann, M., Borschel, L., Bourquin, J.P., Dworzak, M., Klingebiel, T., Lehrnbecher, T., Creutzig, U., Klusmann, J.H. \& Reinhardt, D. (2018) Successes and challenges in the treatment of pediatric acute myeloid leukemia: a retrospective analysis of the AML-BFM trials from 1987 to 2012. Leukemia, 32, 2167-2177.

Roux, C., Tifratene, K., Socie, G., Galambrun, C., Bertrand, Y., Rialland, F., Jubert, C., Pochon, C. Paillard, C., Sirvent, A., Nelken, B., Vannier, J.P., Freycon, C., Beguin, Y., Raus, N., YakoubAgha, I., Mohty, M., Dalle, J.H., Michel, G., Pradier, C., Peffault De Latour, R. \& Rohrlich, P.S (2017) Outcome after failure of allogeneic hematopoietic stem cell transplantation in children with acute leukemia: a study by the societe Francophone de greffe de moelle et de therapie cellulaire (SFGM-TC). Bone Marrow Transplantation, 52, 678-682.

Ruutu, T., de Wreede, L.C., van Biezen, A., Brand, R., Mohty, M., Dreger, P., Duarte, R., Peters, C., Garderet, L., Schonland, S., Gratwohl, A., Niederwieser, D., de Witte, T., Kroger, N. \& European Society for Bone Marrow Transplantation (2015) Second allogeneic transplantation for relapse of malignant disease: retrospective analysis of outcome and predictive factors by the EBMT. Bone Marrow Transplantation, 50, 1542-1550.

Yaniv, I., Krauss, A.C., Beohou, E., Dalissier, A., Corbacioglu, S., Zecca, M., Afanasyev, B.V., Berger, M., Diaz, M.A., Kalwak, K., Sedlacek, P., Varotto, S., Peters, C. \& Bader, P. (2018) Second hematopoietic stem cell transplantation for post-transplantation relapsed acute leukemia in children: a retrospective EBMT-PDWP study. Biology of Blood and Marrow Transplantation: Journal of the American Society for Blood and Marrow Transplantation, 24, 1629-1642. 